6 Rowe JW, Kahn RL. Successful aging. Gerontologist 1997; 37: 433-40.

7 Jeste DV, Harris JC. Wisdom - a neuroscience perspective. JAMA 2010; 304 1602-3.

8 Strawbridge WJ, Wallhagen MI, Cohen RD. Successful aging and well-being: self-rated compared with Rowe and Kahn. Gerontologist 2002; 42: 727-33
9 Vahia IV, Thompson WK, Depp CA, Allison M, Jeste DV. Developing a dimensional model for successful cognitive and emotional aging. Int Psychogeriatr 2012; 24: 515-23.

10 Duckworth AL, Steen TA, Seligman MEP. Positive psychology in clinical practice. Annu Rev Clin Psychol 2005; 1: 629-51.

11 Charney DS. Psychobiological mechanisms of resilience and vulnerability: implications for successful adaptation to extreme stress. Am J Psychiatry 2004; 161: 195-216

\title{
reflections
}

\section{Karl Jaspers, General Psychopathology}

\section{David Goldberg}

In my first months as a trainee at the Maudsley we met weekly with a genial tutor who took us through the whole of General Psychopathology, 50 to 100 pages at a time. Although this seemed an unimaginative way of organising what were optimistically called 'tutorials', the experience has stood me in good stead. Indeed, in work I have recently done in connection with the revision of both the ICD and the DSM, I had Jaspers' aphorism constantly in mind: 'classification . . . has only a provisional value. It is a fiction which will discharge its function if it proves to be the most apt for the time'.

Karl Jaspers qualified in medicine at the age of 25. After 4 short years in clinical psychiatry, at the age of 30 , he wrote General Psychopathology (Allgemeine Psychopathologie), which is indeed the book on which his reputation as a psychiatrist rests.

This starts with what is still the best description of phenomena of abnormal psychological functioning. In making diagnostic assessments, we should be guided by the form, rather than the content of symptoms. His fundamental distinction was between understanding and explaining in thinking about mental disorders. As working clinicians, we are concerned to understand the patient's experiences, but such understanding is necessarily a limited undertaking. The more information we accumulate about an individual patient, the fewer are the possible meanings we can assign to particular experiences - with understanding, there are limits everywhere. In any given case, the judgement of whether a meaningful connection is real does not depend on its selfevident character alone, but depends primarily on tangible facts - about the patient's culture, his acts and way of life, and his underlying personality. The fewer the data, the more interpretations are possible, and the less we understand. Explaining, on the other hand, is the way knowledge advances in psychiatry, and advances by accounting for one set of phenomena in terms of another set of observations. It is essentially an unlimited procedure.

Jaspers considered that only psychological disorders accompanying known cerebral pathology were true disease entities, and within the psychoses 'the diagnosis has no specific alternative character. Either it is clear as a whole or the differential diagnosis over details determines nothing'.

'If the reader tries to get hold of the entity involved, he will find that it melts away even as he looks at it. The question of what underlies all phenomena in general used to be answered in the old days by the notion of evil spirits. These later turned into disease entities that could be found by empirical investigation. They have proved themselves however to be mere ideas'.

'We have intuitions of a whole which we call schizophrenia but we do not grasp it; instead we enumerate a vast number of particulars or simply say "ununderstandable", while each of us only comprehends the whole from his own experience of actual contact with such patients'.

Nor did he have much time for precise diagnoses in the field of neuroses and personality disorders (psychopathien): 'it is difficult to bring diagnostic order ... into shifting phenomena which continually keep merging into one another ... there is no sharp dividing line between types nor is there a decisive borderline between what is healthy and what is not. A diagnosis remains typological and multi-dimensional including a delineation of the type of personality'.

After leaving psychiatry Jaspers became one of the founding fathers of existential philosophy.

A series of 'Reflections on Karl Jaspers' commemorates the centenary of the first publication of his Allgemeine Psychopathologie in 1913. 